\title{
Development and bench tests of a programmable logic controller "Simens Logo" based control system of an electromagnetic hammer for destruction of boulders
}

\author{
Konstantin Vassin ${ }^{12^{*}}$, Yerik Yedygenov ${ }^{1}$, Nakhypbek Aldiyarov ${ }^{2}$ and Vladimir Voronin ${ }^{3}$ \\ ${ }^{1}$ D.A. Kunayev Mining Institute, Almaty, Kazakhstan \\ ${ }^{2}$ Satbayev University, Almaty, Kazakhstan \\ ${ }^{3}$ Pacific National University, Khabarovsk, Russia
}

\begin{abstract}
The development a programmable logic controller (PLC) based control system of electromagnetic hammer for destruction of boulders is described in the article. During bench tests of the control system, the possibility of using a PLC "Simens Logo" for multi-mode control of electromagnetic actuators of hammer is demonstrated.
\end{abstract}

\section{Introduction}

Currently, the mining industry is experiencing difficulties associated with the increasing complexity of mining and geological conditions: the transition to the ore production at greater depths; the need to process low-grade ore; an increase in the proportion of ore extraction from thin and very thin seams, which requires selective mining of valuable ores. Under these conditions, the efficiency of the widely used drilling and blasting operations is reduced, and it is necessary to develop alternative methods and technologies of blastless mining.

Blastless methods of rock breaking - among which it is possible to distinguish the hydraulic [1-4] and the most widely used mechanical methods [5, 6] - ensure the loss reduction due to a more thorough development of the areas where the rock contacts the ore, and ensure selective ore mining, environmental cleanliness, performing mining operations without compromising safety in nearby buildings, settlements, and transportation networks.

Impact machines that work effectively in technological processes requiring powerful concentrated energy effects, namely, in the mining and construction industry, have received widespread use $[7,8]$.

Impact machines are able to excavate minerals with a significant reduction in their losses, to maintain continuous monitoring of the mined ore quality as well as to reduce the adverse environmental impact of mining operations, and to increase the mining safety. Therefore, presently, the works on improving technological and functional capabilities of impact machines and on expanding the field of application of blastless technologies become more and more urgent.

\footnotetext{
* Corresponding author: kvas2500@mail.ru
} 
Currently, to break rocks or perform road construction operations, the hydraulic hammers are used as an impactor attachment on an excavator, performance of which, as the practice shows, is reduced at low temperatures, and they are designed to break rocks of a certain hardness.

Another direction in the development of impact machines is the creation of electromagnetic hammers that use the energy of a magnetic field as a driving force [9-11].

In "Destruction and delivery of rock" laboratory of the D.A. Kunaev Mining Institute (Almaty, Kazakhstan), by targeted funding for 2018-2020 under the subprogram "Technological modernization of mining production based on the transition to the digital economy "is being carried out the work on the topic "Develop technologies and mining equipment for the non-explosive extraction of solid minerals".

In 2020, to develop a technological scheme of secondary crushing of rocks using an electromagnetic hammer with digital control is planned. The author, $\mathrm{PhD}$ student of Satbayev University and co-authors, developed an automated control system (ACS) of an electromagnetic hammer (EMH) based on a programmable logic controller (PLC) to implement a digital control system for an electromagnetic hammer.

The device and principle of operation of EMH are described in [11, 12, 14]. These papers show that the movable part of the EMH is driven by linear electromagnetic idling and working actuators. These actuators are armored solenoids with free run out of armatures. Turning on and off these electromagnetic actuators is carried out using the electronic control unit. The first versions of the electronic control unit are described in papers [12, 13]. These papers show that the 155 series of digital logic circuits were used to generate control signals.

The main goal of the EMH automated control system being developed is to increase the reliability and efficiency of rock destruction. To increase the destruction efficiency, it was proposed to use a multi-mode method for controlling electromagnetic actuators, which inevitably leads to a complication of the circuitry. After familiarizing ourselves with the capabilities of the "Simens Logo" PLC in the educational and experimental laboratory of modern automation equipment Siemens from the Department of Automation and Systems Engineering of the Pacific National University (Khabarovsk, Russia), it was decided to use it to control electromagnetic actuators. This will allow, using the high reliability of the PLC, to load into it a control program containing up to 400 logic elements.

\section{Theoretical description of the developed EMH control system}

The electromagnetic hammer (hereinafter the EMH) is designed as a medium-class hammer with a 6,000 J impact energy and manufactured at JSC "S.M. Kirov Machine-building Plant" funded by the innovation grant from JSC "National Agency for Technological Development" (2016-2019).

Figure 1 shows the functional diagram of the developed automated control system of the electromagnetic hammer. 


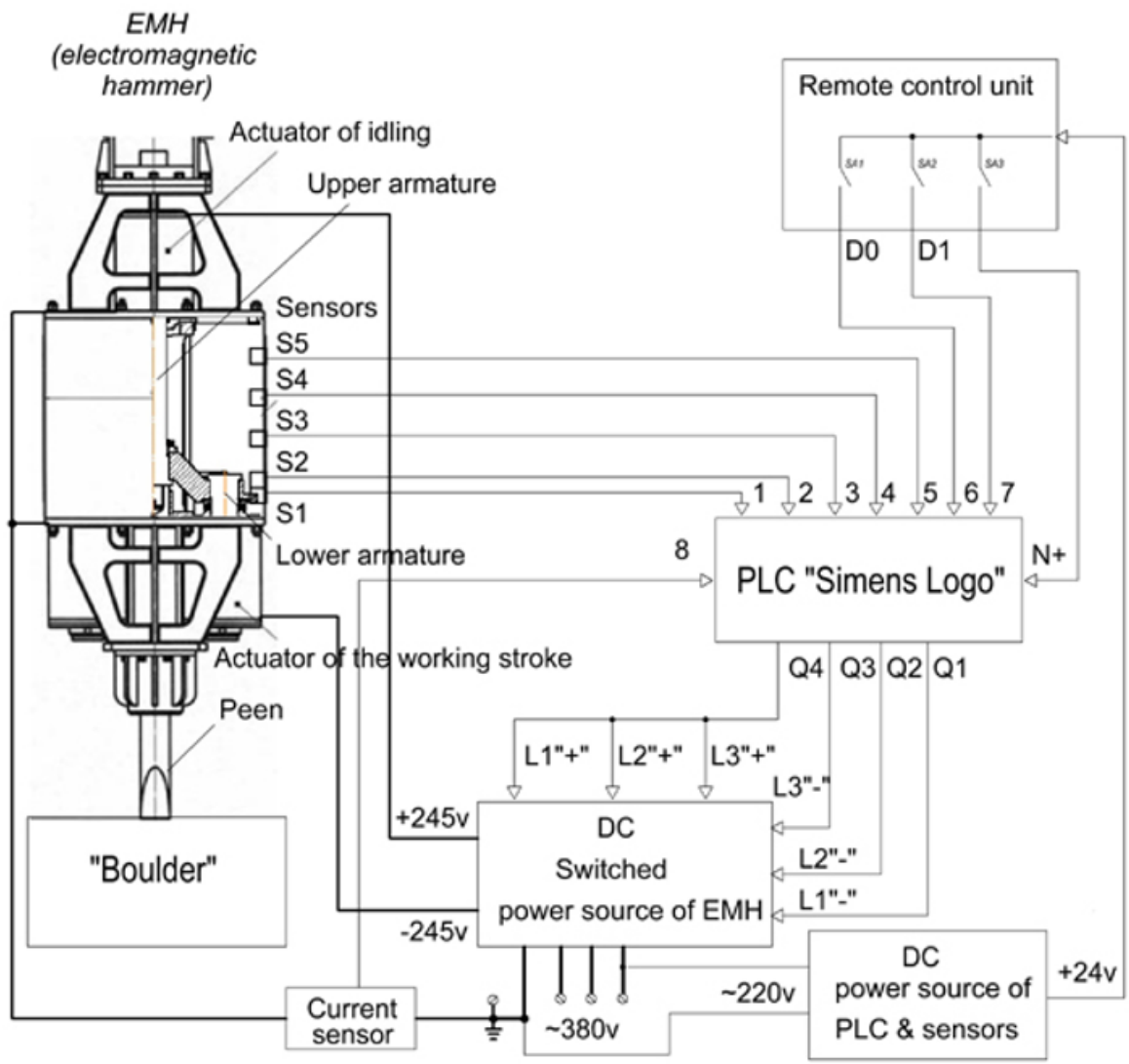

Fig. 1. Automated control system for electromagnetic hammer.

The device and the principle of operation of the electromagnetic hammer are described in paper [4]. The work of EMH consists in the sequential execution of the following stages:

1. Platoon - raising the striking part to accumulate potential energy;

2. Working stroke - fall - transformation of potential energy into kinetic energy plus acceleration to impact speed;

3. Impact - the interaction of the striking part through the striker with the rock - impact speed plus the duration of the impact.

All three stages of EMH work make up the impact cycle. Thus, for the implementation of multimode control, it is necessary to provide automated regulation of the technological parameters of each stage. Electric power supply of electromagnetic actuators is carried out by a controlled DC source, which allows controlling the rectification of positive and negative half-waves of each phase of a three-phase industrial main. The position of the moving part of the EMH is monitored by five inductive sensors S1-S5. The signals from the S4 or S5 sensors at the "Platoon" stage allow lifting the moving part to the appropriate height using the electric energy of all three phases of the positive halfwave, and at the "Working stroke" stage, according to the signals from the S5, S4 and S3 sensors, turn on respectively L1, L2 and L3 phases of the negative half-wave, thereby carrying out a sequential acceleration. Sensors S2 and S1 are operating in the "Impact" stage. According to the signal from the S2 sensor, which is at the height of the impact of the moving part on the striker, a one second delay is triggered for switching from the "Working stroke" stage to the "Platoon" stage. This allows at the moment of impact to press both the movable part and the striker against the rock to be destroyed to ensure maximum breaking force. If during this second the rock is destroyed, then the striker, together with the moving part, falls through and the S1 sensor is triggered, at the signal of which the ACS deenergizes the EMH. If the rock is not destroyed by impact, a new impact cycle is started. The activation of the EMH is carried out by the signal of the $\mathrm{S} 2$ sensor, when the EMH is pressed against 
the destroyed rock. To control overcurrents in the developed ACS, a "Current sensor" is provided, according to the signal of which, in case of exceeding the set values, the ACS de-energizes the EMH. The brain of the ACS EMH is the "Simens Logo" PLC, which processes the signals from the S1-S5 sensors and "Current sensor", and generates control signals to the power source for the hammer electromagnetic actuators. "Simens Logo" PLC and sensors are powered by a separate 24 volt power supply.

The developed version of ACS EMH provides four modes of rock destruction cycles control:

No. $1(00)$ - Ascent to the S5 sensor and thrust with full power;

No. 2 (01) - Ascent to the S4 sensor and thrust with full power;

No. 3 (10) - Ascent to the S5 sensor and thrust with increased power;

No. 4 (11) - Ascent to the S4 sensor and free fall without thrust by the actuators.

The control modes of the ACS are set using a remote control unit with a digital code.

The LOGO! Soft Comfort was used to create and download a circuit program to the "Simens Logo" PLC.

Figure 2 shows a unit diagram of the circuit program of the PLC "Simens Logo".

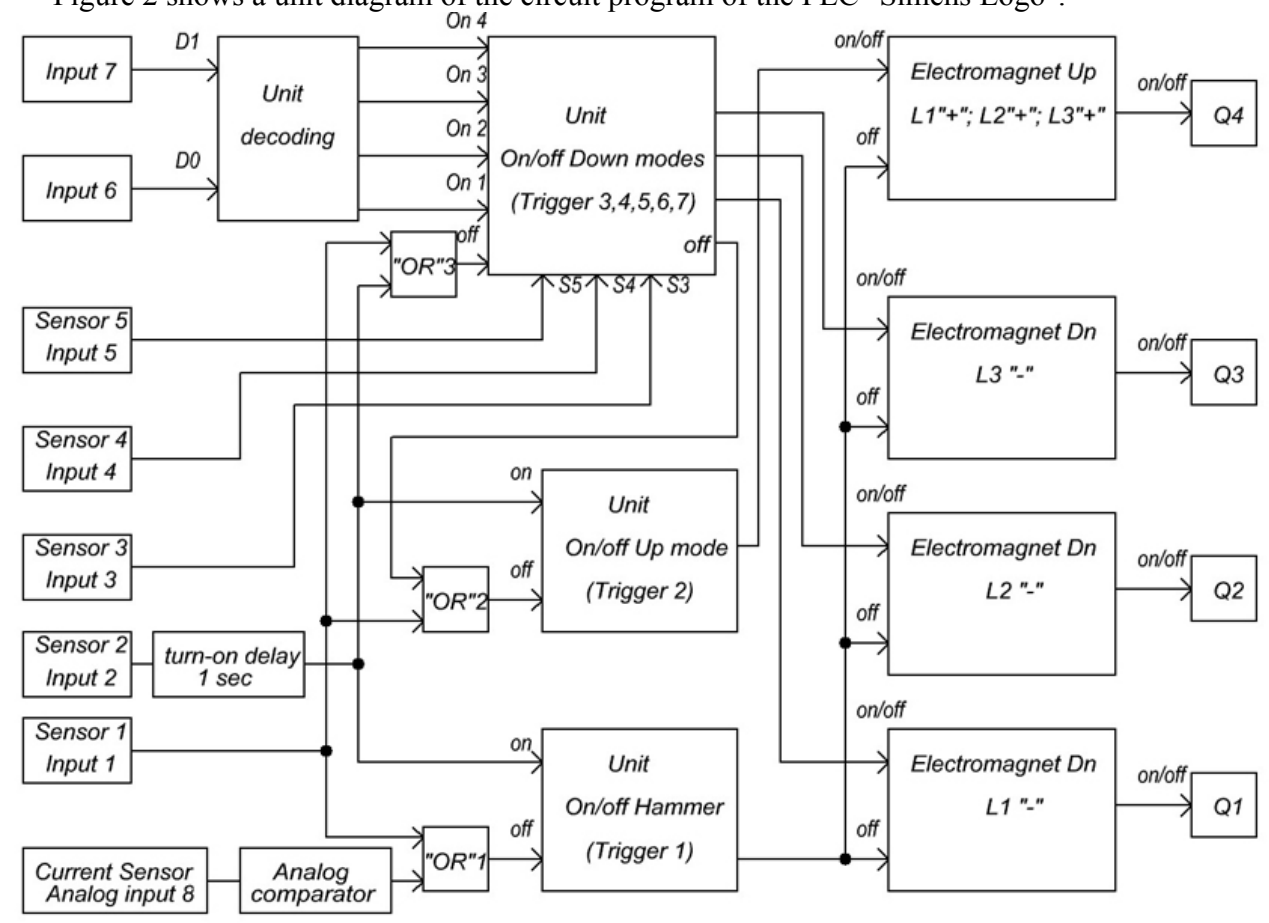

Fig. 2. Unit diagram of the circuit program of the PLC "Simens Logo".

This ACS logic unit diagram operates on all PLC input and output signals. One analog and seven digital signals are supplied to the PLC input. The analog signal "AnalogInput8" is supplied from the "Current sensor" of the both idling and working actuators. When the currents exceed the limit values, the logical comparator through the "OR" 1 element switches the trigger 1 to the off state, which leads to the automatic shutdown of all transistor outputs of the PLC Q1 - Q4. The signal at the digital input "Input 1" from the S1 sensor through the "OR" 1 element switches the trigger 1 off, which leads to automatic shutdown of all transistor outputs of the PLC Q1 - Q4. The signal at the digital input "Input 2 " from the S2 sensor through the one second delay element switches the trigger 2 into the on state, which automatically turns on the transistor output of the PLC Q4 and automatically turns off all the transistor outputs of the PLC Q1 - Q3. The signals at the digital inputs "Input 3,4,5" from the sensors S3, S4, S5 switch triggers 3,4,5,6,7 into the on state depending on the control mode, which leads to automatic shutdown, through trigger 2 , of the transistor PLC output Q4 and to automatically turn on the transistor outputs of the 
PLC Q1 - Q3, depending on the control mode. The signals at the digital inputs "Input 6, 7" from the remote control, through the decoding unit, set the ACS control mode.

In mode No. 1, the transistor output Q4 on the signal of trigger 2 connects three positive phases of the mains to the idle actuator, and the magnetic force raises the moving part of the EMH until the S5 sensor is triggered. Transistor outputs Q1, Q2 and Q3, on the signal of trigger 4 , connects three negative phases of the mains to the working stroke actuators, providing full thrust of the moving part to strike the destructible rock.

In mode No. 2, the transistor output Q4 on the signal of trigger 2 connects three positive phases of the mains to the idle actuator, and the magnetic force raises the moving part of the EMH until the sensor S4 is triggered. Transistor outputs Q1, Q2 and Q3, on the signal of trigger 3, connects three negative phases of the mains to the working stroke actuators, providing full thrust of the moving part for impacting the destructible rock.

In mode No. 3, the transistor output Q4 on the signal of trigger 2 connects three positive phases of the mains to the idle actuator, and the magnetic force raises the moving part of the EMH until the sensor S5 is triggered. Transistor outputs Q1, Q2 and Q3, according to the signals of triggers $5,6,7$, connect the negative phases of the mains to the actuators of the working stroke separately, providing a sequential acceleration of the moving part to strike the destructible rock.

In mode No. 4, the transistor output Q4, on the signal of trigger 2, connects three positive phases of the mains to the idle actuator, and the magnetic force raises the moving part of the EMH until the S4 sensor is triggered. Transistor outputs Q1, Q2 and Q3 are not turned on in this mode, and the moving part falls in free fall to strike against the destructible rock.

\section{The results of bench tests of developed the automated control system and discussions}

To test the circuit program and check the possibility of using the programmable logic controller "Simens Logo" for multi-mode control of electromagnetic actuators of the hammer, a special stand was developed and manufactured. To simulate the current sensor, the potentiometer R13 is used. To simulate the position sensors of the moving part, the buttons SB1-SB5 are used. To indicate the input and output signals, the HL1-HL11 LEDs are used. Figure 3 shows an electrical circuit diagram and a photo of the ACS stand.
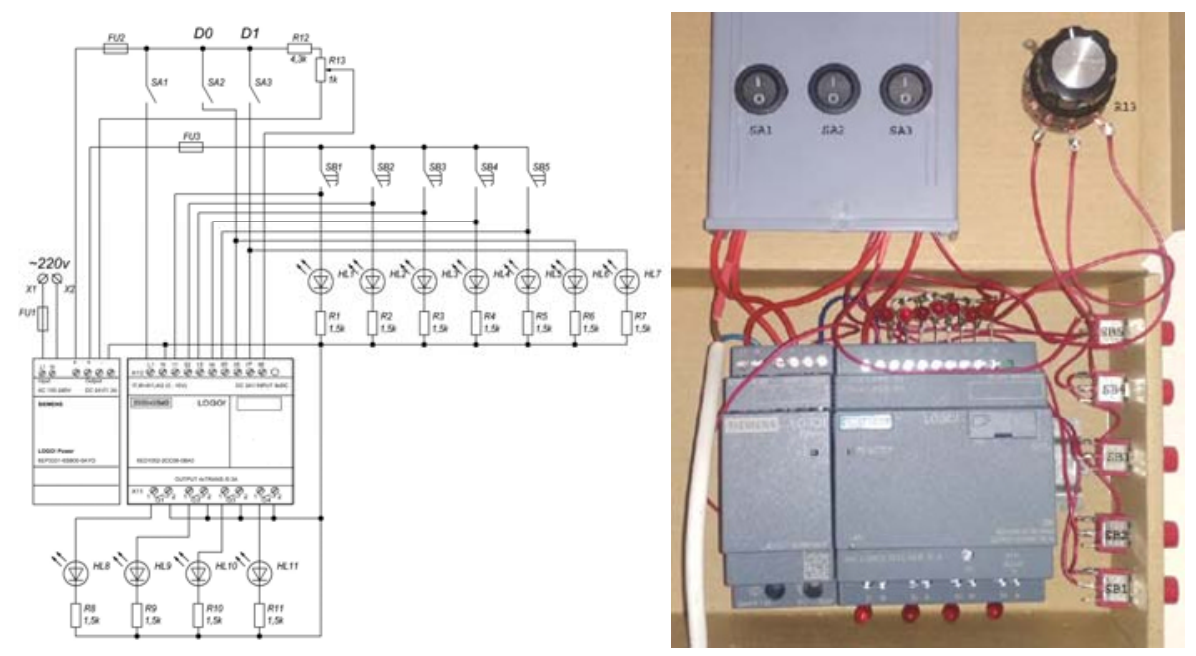

Fig. 3. Electrical circuit diagram and photo of the ACS stand. 
Figures 4, 5, 6, 7 show the timing diagrams of the ACS stand in control modes 1, 2, 3, 4. After the first cycle, rock destruction was simulated and sensor S1 was triggered. Further, a new cycle shows the impact process without destruction.

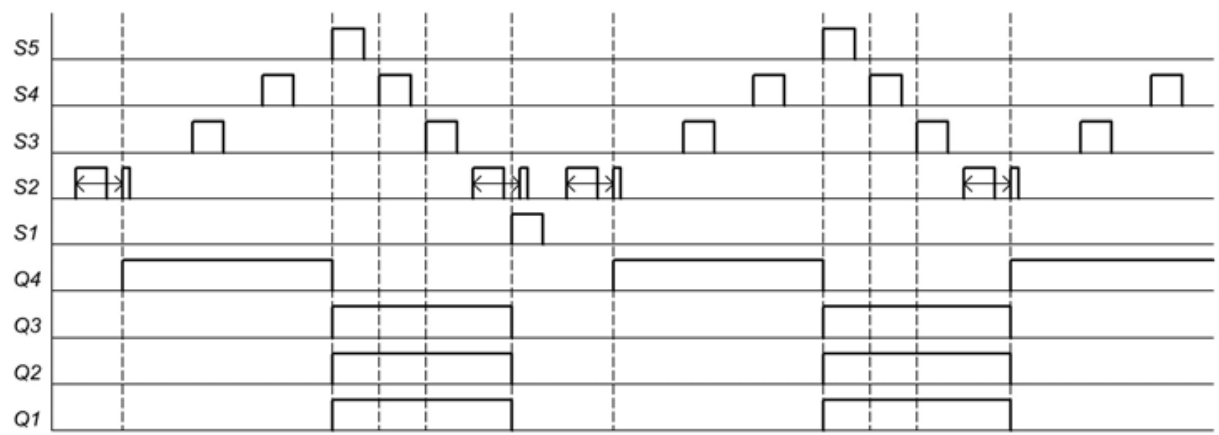

Fig. 4. Timing diagram of the ACS operation mode No. 1 (00) Ascent to the upper position sensor S5 and thrust with full power.

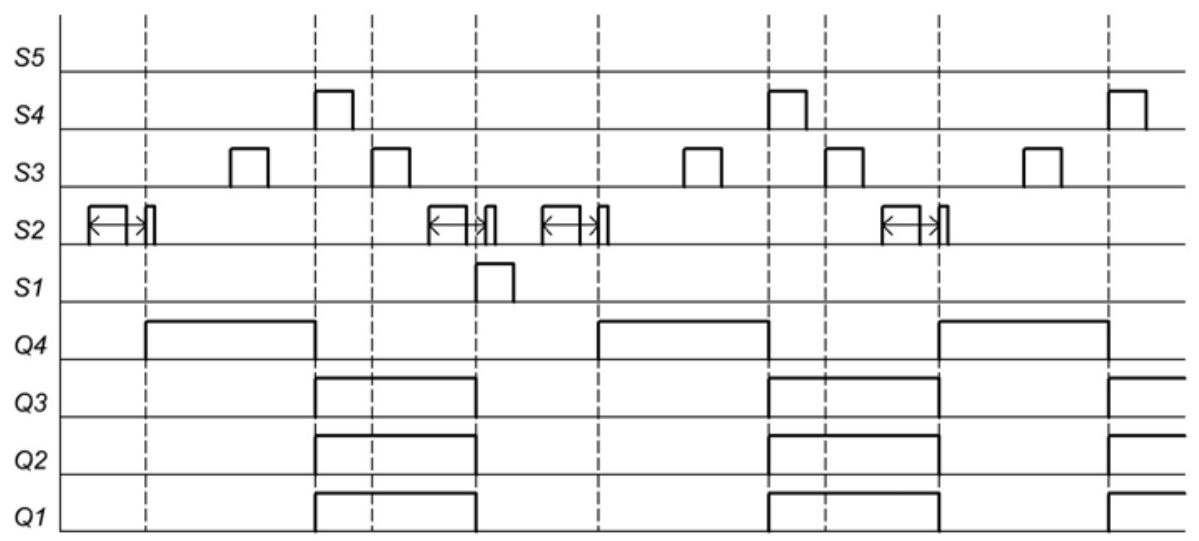

Fig. 5. Timing diagram of the ACS operating mode No. 2 (01) - Ascent to the position sensor S4 and thrust with full power.

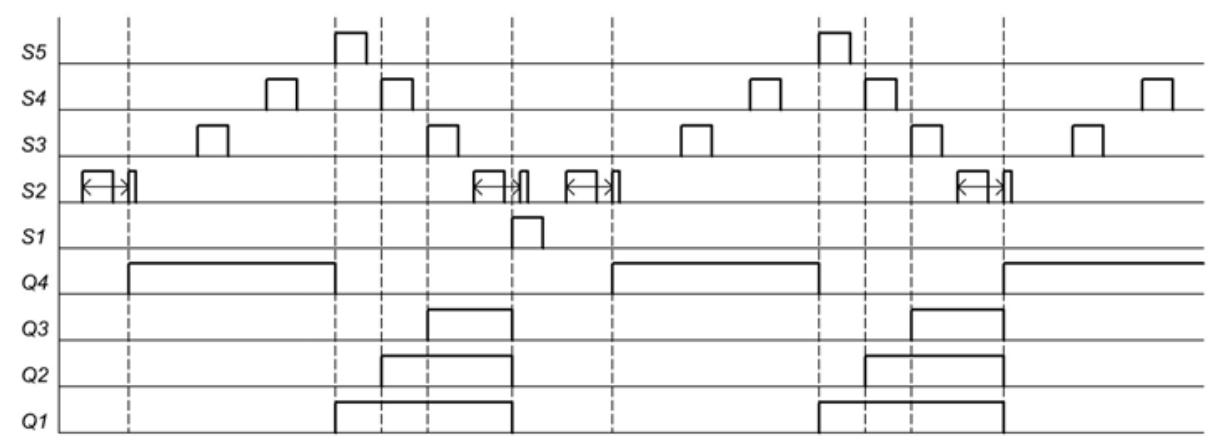

Fig. 6. Timing diagram of the ACS operation mode No. 3 (10) - Ascent to the position sensor S5 and thrust with sequentially increased power. 


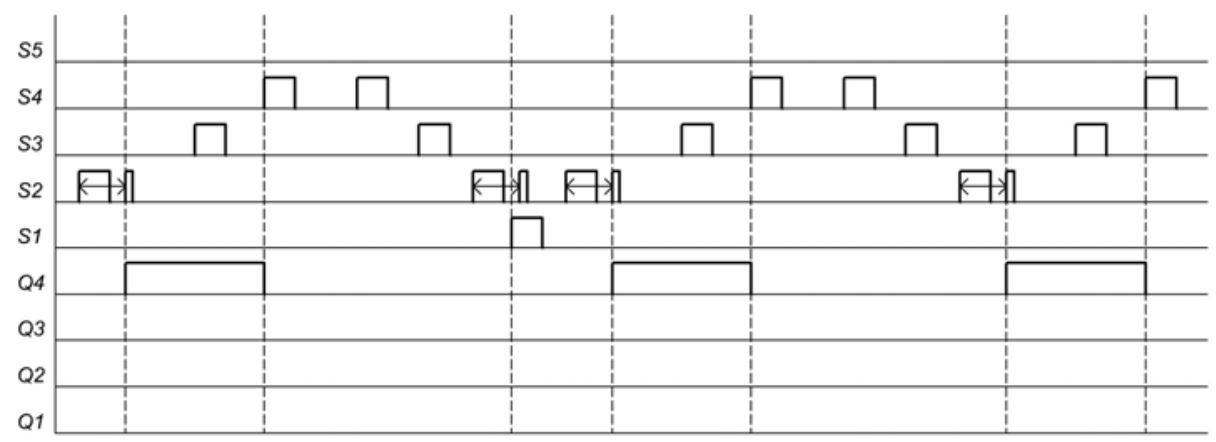

Fig. 7. Timing diagram of the ACS operation mode No. 4 (11) - Ascent to the position sensor S4 and free fall without thrust by electromagnets.

Based on the results of bench tests of the automated control system, the operability of the circuit program and the possibility of using the programmable logic controller "Simens Logo" for multi-mode control of electromagnetic hammer actuators were checked.

\section{Conclusions}

Therefore, the developed automated control system for an electromagnetic hammer based on a PLC makes it possible to control the technological modes of destruction of boulders. The use of the programmable logic controller "Simens Logo", due to the possibility of reprogramming, allows you to improve and complicate the multimodal control without additional financial costs.

The experience of creation and test results showed that:

- In terms of design, the EMH is quite simple to manufacture, since there are no parts that must be produced with high accuracy, there are no strict requirements in terms of fit, and the manufacturing does not require deficit materials and special equipment;

- The electromagnetic hammer can steadily work at low temperatures and it is possible to regulate the energy of a single blow in a wide range;

- The electromagnetic hammers can be used by mining and construction enterprises in conditions of a sharply continental climate.

Acknowledgement. The work was performed in the framework of the Target Financing Program No BR05236712 "Technological modernization of mining production based on the transition to the digital economy" (2018-2020) of the Ministry of Education and Science of the Republic of Kazakhstan.

\section{References}

1. Y. Melamed, A. Kiselev, M. Gelfgat, D.Dreesen and B.James, J. Energy Resour. Technol, 122(1), 1-7 (1999)

2. J.R. Tang, ZL Ge., L.U. Xia and B.W. Wang, Journal of Mining \& Safety Engineering, 30(4), 621-627 (2013)

3. H. Jokiniemi, Publications committee of XIV ICSMFE, 1077- 1080 (1997)

4. H. Andersson, K. Simonsson end D. Hilding, Advances in engineering software, 131, 103-115 (2019)

5. P. Berry, G.A. Delengini and S. Fabbri, Mine Planning and Equipment Selection 2000 Ballkema, Rotterdam, ISSN 9058091783, 521-526 (2000) 
6. M. Dolipsky, M. Jaszczuk, P. Sobota, E. Kusak, V.M. Kurek, Mine Planning and Equipment Selection 2000 Ballkema, Rotterdam, ISSN 905809 1783, 541-546 (2000)

7. T. Deniz end H. Shahabedin, Tunn Undergr Sp Tech, 59, 37-47 (2016)

8. I. Ocak, S.E. Seker and J. Rostami, Tunnelling and underground space technology, 80, 269 - 276 (2018)

9. L.C. Lemos Junior, J.J. Silva and J.S. Rocha Neto, Joint imeko TC1-TC7-TC13 symposium: measurement science challenges in natural and social sciences, 1044, UNSP012029 (2017)

10. T. Wu, Y.R. Tang and S.W. Tang, Iet elektric powerapplications, 11(I)9, 1556-1565 (2017)

11. Ye.K Yedygenov, V. Lyashkov, International Symposium on planning of mining and selection of equipment of MPES 2011, Almata, 1002-1012 (2011)

12. Vasin Konstantin; Yedygenov Yerik; Aldiyarov Nakhypbek; Voronin Vladimir, E3S Web of Conferences, 56, 01024, e-ISSN: 2267-1242, DOI: https://doi.org/10.1051/e3sconf/20185601024

13. K. Vasin, Ye. Yedygenov, N. Aldiyarov, V. Voronin, 2019 International MultiConference on Industrial Engineering and Modern Technologies (FarEastCon) https://ieeexplore.ieee.org/document/8934020, DOI: 10.1109/FarEastCon.2019.8934020

14. Ye.K. Yedygenov, K.A. Vasin, Mining Informational and Analytical Bulletin, 5, 80-90 (2020), DOI: 10.25018/0236-1493-2020-5-0-80-90 